\title{
Benthic Foraminiferal Census Data from Louisiana Continental Shelf Cores, Gulf of Mexico
}

Series 2008-1348 



\section{Benthic Foraminiferal Census Data from Louisiana Continental Shelf Cores, Gulf of Mexico}

By Lisa E. Osterman, Wendy S. Kelly, and John P. Ricardo

Open-File Report 2008-1348

U.S. Department of the Interior

U.S. Geological Survey 


\section{U.S. Department of the Interior \\ KEN SALAZAR Secretary \\ U.S. Geological Survey \\ Suzette M. Kimball, Acting Director}

U.S. Geological Survey, Reston, Virginia: 2009

For more information on the USGS - the Federal source for science about the Earth, its natural and living resources, natural hazards, and the environment, visit http://www.usgs.gov or call 1-888-ASK-USGS

For an overview of USGS information products, including maps, imagery, and publications, visit http://www.usgs.gov/pubprod

To order this and other USGS information products, visit http://store.usgs.gov

Any use of trade, product, or firm names is for descriptive purposes only and does not imply endorsement by the U.S. Government.

Although this report is in the public domain, permission must be secured from the individual copyright owners to reproduce any copyrighted materials contained within this report.

Suggested citation:

Osterman, L.E., Kelly, W.S., and Ricardo, J.P., 2009, Benthic Foraminiferal Census Data from Louisiana Continental Shelf Cores, Gulf of Mexico: U.S. Geological Survey Open-File Report 2008-1348, 16 p. 


\section{Contents}

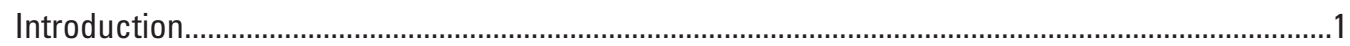

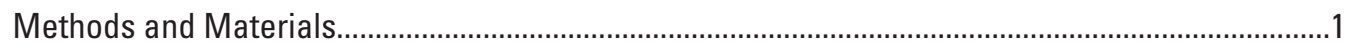

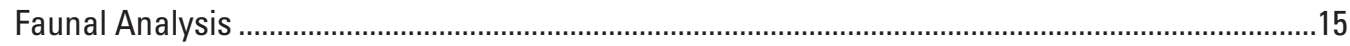

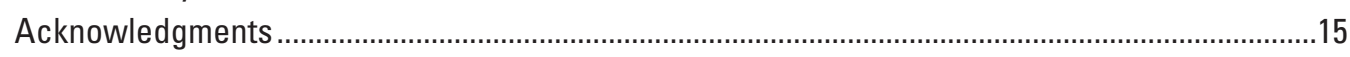

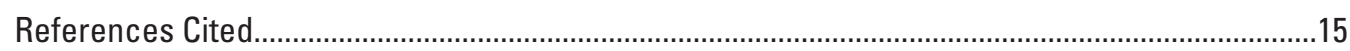

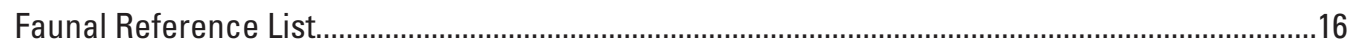

\section{Figure}

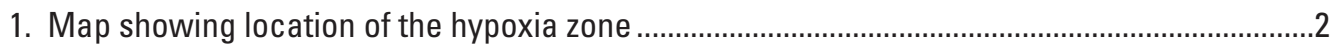

\section{Tables}

1. Longitude and latitude of Louisiana Shelf cores, Gulf of Mexico $\ldots \ldots \ldots \ldots \ldots \ldots \ldots \ldots \ldots \ldots \ldots \ldots \ldots \ldots . . .2$

2-11. Benthic foraminiferal data from core:

2. MRJ03-2BC, Louisiana Shelf, Gulf of Mexico ………..................................................

3. MRJ03-3BC, Louisiana Shelf, Gulf of Mexico .............................................................

4. MRD05-6BC, Louisiana Shelf, Gulf of Mexico .............................................................

5. MRD05-6GC, Louisiana Shelf, Gulf of Mexico .......................................................... 6-8

6. PE0609BC, Louisiana Shelf, Gulf of Mexico …………………………………......

7. MCH-11BC, Louisiana Shelf, Gulf of Mexico

8. MCH-33BC, Louisiana Shelf, Gulf of Mexico

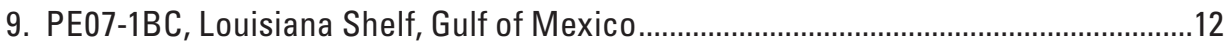

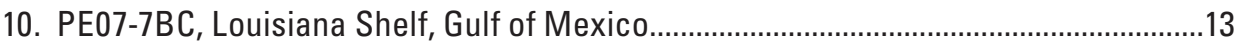

11. PE07-8BC, Louisiana Shelf, Gulf of Mexico …………….........................................14 


\section{Conversion Factors, Acronyms, and Abbreviations}

\begin{tabular}{rll} 
Multiply & \multicolumn{1}{c}{ By } & To obtain \\
micrometer $(\mu \mathrm{m})$ & 0.0000394 & inch (in.) \\
centimeter $(\mathrm{cm})$ & 0.3937 & inch (in.) \\
meter $(\mathrm{m})$ & 3.281 & foot (ft) \\
kilometer $(\mathrm{km})$ & 0.6214 & mile (mi) \\
$\operatorname{liter}(\mathrm{L})$ & 33.82 & ounce, fluid (fl. oz) \\
$\operatorname{milliter}(\mathrm{mL})$ & 0.03382 & ounce, fluid (fl. oz) \\
cubic centimeter $\left(\mathrm{cm}{ }^{3}\right)$ & 0.06102 & cubic inch (in $\left.{ }^{3}\right)$ \\
$\operatorname{gram}(\mathrm{g})$ & 0.03527 & ounce, avoirdupos (oz) \\
$\operatorname{Celsius~}\left({ }^{\circ} \mathrm{C}\right)$ & ${ }^{\circ} \mathrm{F}=\left(1.8 \times{ }^{\circ} \mathrm{C}\right)+32$ & Fahrenheit $\left({ }^{\circ} \mathrm{F}\right)$ \\
\hline
\end{tabular}




\title{
Benthic Foraminiferal Census Data from Louisiana Continental Shelf Cores, Gulf of Mexico
}

\author{
By Lisa E. Osterman, Wendy S. Kelly, and John P. Ricardo
}

\section{Introduction}

An area of oxygen-depleted bottom- and subsurfacewater (hypoxia $=$ dissolved oxygen $<2 \mathrm{mg} / \mathrm{L}^{-1}$ ) occurs seasonally on the Louisiana Shelf near the Mississippi River (fig. 1). The area of hypoxia, also known as the "dead zone," forms when spring and early summer freshwater flow from the Mississippi River supplies a large amount of nutrients to the shelf while creating a freshwater lens, or cap, above the shelf water. The excess nutrients cause phytoplankton blooms in the shallow shelf water. After the bloom ceases, the organic material sinks in the water column and uses up oxygen during decomposition. Thus, the subsurface waters become oxygen depleted. The seasonal dead zone exists until a reduction in freshwater flow, or overturning by storms, allows mixing of the water column to restore normal oxygen conditions (Rabalais and others, 1994, 1996; Rabalais, 2002).

Since systematic measurement of the extent of the dead zone began in 1985, the overall pattern indicates that the area of the dead zone is increasing (Rabalais and Turner, 2001; Turner and others, 2005). Several studies have concluded that the expansion of the Louisiana Shelf dead zone is related to increased nutrients (primarily nitrogen, but possibly also phosphorous) in the Mississippi River drainage basin and is responsible for the degradation of Gulf of Mexico marine habitats (Goolsby and others, 2001). This paper presents the benthic foraminiferal data from 10 sediment cores collected from the Continental Shelf of Louisiana (table 1), obtained as part of an initiative to investigate the geographic and temporal extent of hypoxia prior to 1985 in the Gulf of Mexico (fig. 1).
Benthic foraminifers provide a method to track the development of hypoxia prior to 1985 (Blackwelder and others, 1996; Sen Gupta and others, 1996). Previous work (Osterman, 2003) has shown statistically that the relative occurrence of three low-oxygen-tolerant species represents the modern seasonal Louisiana hypoxia zone (fig. 1). The cumulative percentage of these three species ( $\%$ Pseudononion atlanticum $+\%$ Epistominella vitrea, $+\%$ Buliminella morgani $=$ PEB index of hypoxia) provides a way to investigate fluctuation in paleohypoxia. Interpretation of some of these cores is provided in Osterman and others (2005), Osterman and others (2008a,b), and Swarzenski and others (2008). Our hypothesis is that the increased relative abundance of PEB species in dated sediment cores accurately tracks past seasonal low-oxygen conditions on the Louisiana Shelf.

\section{Methods and Materials}

The cores were collected between 20 and $231 \mathrm{~m}$ water depth (mwd) during five cruises (table 1). Gravity and box cores were collected on the Louisiana Continental Shelf and Slope on the R/V Pelican cruises MRD05 (May 2005), PE06 (July 2006), MCH (April 2007), and PE07 (April 2007). Two box cores were collected on the R/V Longhorn cruise MRJ03 (July 2003). In all cases, the box cores were subsampled using push cores that were extruded and sampled within 1 day. Gravity core MRD05-06 was sectioned and capped after collection. The gravity-core and box-core samples were refrigerated and transported to the U.S. Geological Survey (USGS), 


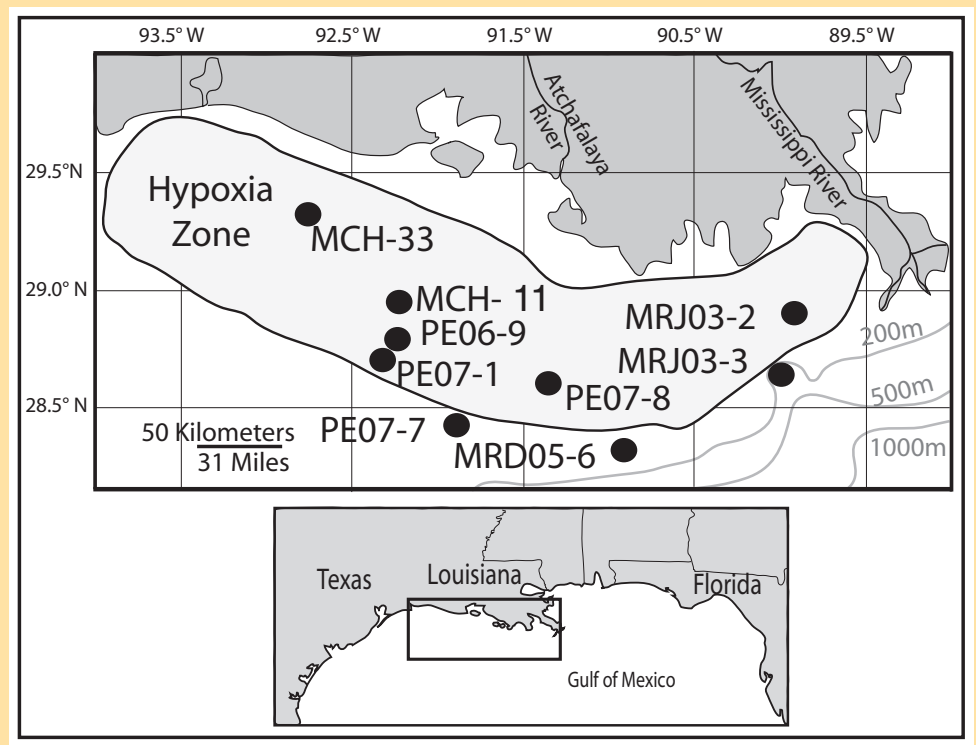

Figure 1. Location of the hypoxia zone as measured by Rabalais and others (1999). Also shown are the locations of the gravity core (MRD05-6) and the nine box cores. Shelf bathymetry is shown in meters of water depth.

Table 1. Longitude and latitude of Louisiana Shelf cores, Gulf of Mexico.

\begin{tabular}{lcccc}
\hline \multicolumn{1}{c}{ Core } & $\begin{array}{c}\text { Latitude "decimal } \\
\text { degrees" }\end{array}$ & $\begin{array}{c}\text { Longitude "decimal } \\
\text { degrees" }\end{array}$ & $\begin{array}{c}\text { Water } \\
\text { depth }(\mathbf{m})\end{array}$ & $\begin{array}{c}\text { Core } \\
\text { length }(\mathbf{c m})\end{array}$ \\
\hline MRJ03-2BC & 28.89033 & 89.89300 & 43 & 52 \\
MRJ03-3BC & 28.62580 & 90.00090 & 231 & 58 \\
\hline MRD05-6BC & 28.27910 & 90.90950 & 65 & 42 \\
MRD05-6GC & 28.27990 & 90.91120 & 65 & 153 \\
\hline PE06-09BC & 28.77500 & 92.38330 & 33 & 18 \\
MCH-11BC & 28.68060 & 92.20960 & 30 & 36 \\
\hline MCH-33BC & 29.19840 & 92.70110 & 20 & 36 \\
PE07-1BC & 28.69972 & 92.39110 & 33 & 38 \\
\hline PE07-7BC & 29.49528 & 91.99722 & 47 & 31.5 \\
PE07-8BC & 28.58300 & 91.36670 & 32 & 37 \\
\hline
\end{tabular}

in St. Petersburg, FL, where the gravity-core sediments in plastic core liners were extruded and sampled within weeks of collection. Each 1-cm slice, of either box- or gravity-core sediment, was divided into three subsamples and stored either frozen in precombusted glass jars (organics), or refrigerated in pre-weighed plastic cups (trace elements and radionuclides) or in plastic bags (foraminifers) for further processing.

The foraminiferal samples were processed at the USGS Foraminiferal Research Laboratory in Reston, VA, before October 2005 and in St. Petersburg, FL, after that date. Each $1-\mathrm{cm}$ sediment sample for faunal analyses was soaked in water with $10 \mathrm{~mL}$ of percent Calgon solution, slowly agitated for up to 1 hour to aid disaggregation, then washed over a stainless steel $63-\mu \mathrm{m}$ sieve. In most cases, the top 8 to 10 samples in each box core were additionally treated with $10 \mathrm{~mL}$ of 8 percent hydrogen peroxide solution to disaggregate organic material. After sieving, the remaining sample was oven dried at $\leq 60{ }^{\circ} \mathrm{C}$, then dry sieved at $125 \mu \mathrm{m}$. The samples were not dried prior to the wet-sieving process; the calculated dry weights were obtained by measuring the moisture content of a small subsample of each sample. More recently, we have measured the wet volume (cubic centimeter) of each sample prior to wet sieving. The calculated dry sample weight, or wet sample volume, is used to calculate the number of forams per gram or cc, respectively (tables 2-11). 
Table 2. Benthic foraminiferal data from core MFJ03-2BC, Louisiana Shelf, Gulf of Mexico.

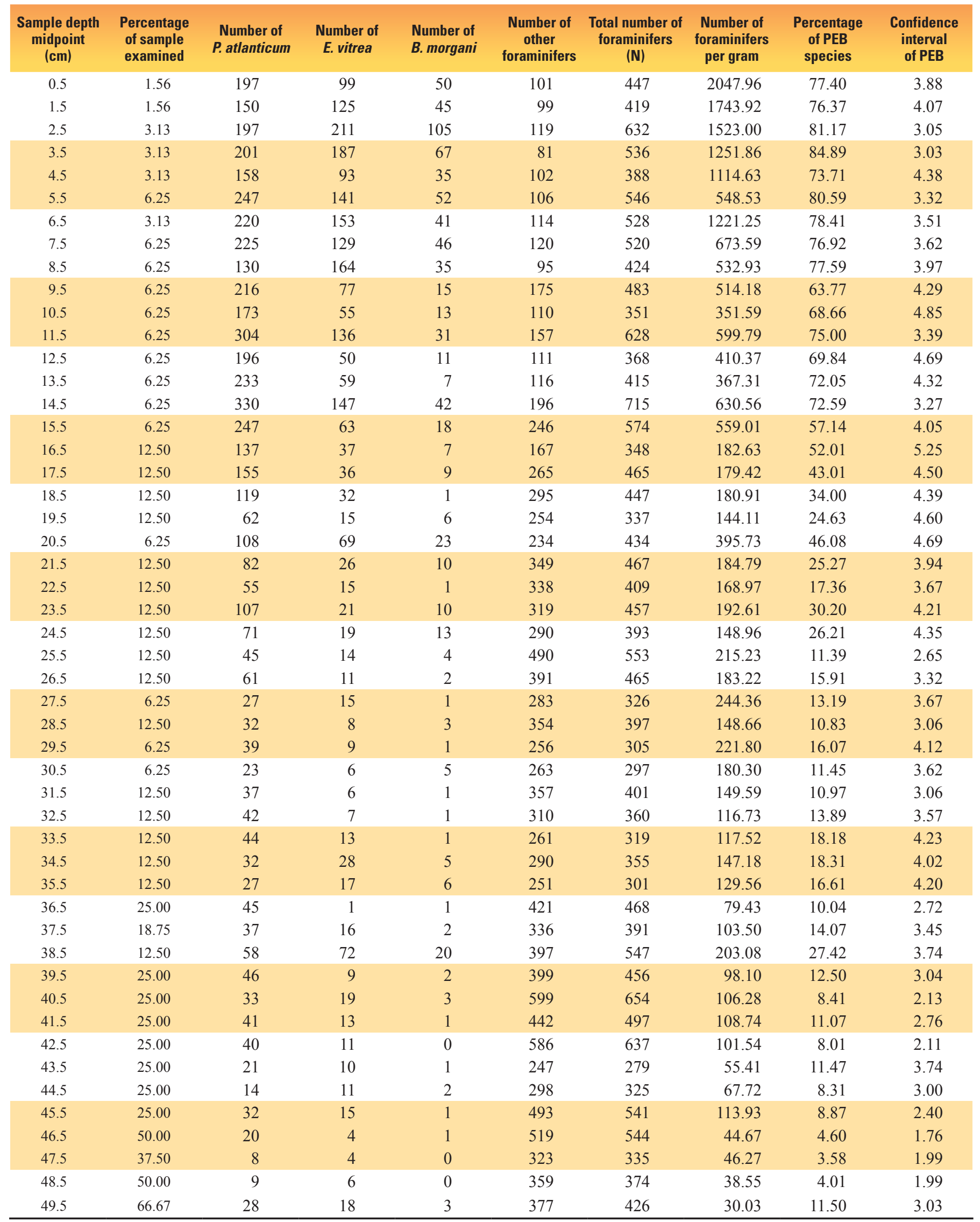


Table 3. Benthic foraminiferal data from core MRJ03-3BC, Louisiana Shelf, Gulf of Mexico.

[See text (Introduction) for definition of PEB; due to extremely rapid sedimentation rate $(1.24 \mathrm{~cm} / \mathrm{yr})$, not all samples were counted]

\begin{tabular}{|c|c|c|c|c|c|c|c|c|c|}
\hline $\begin{array}{l}\text { Sample } \\
\text { depth } \\
\text { midpoint } \\
\text { (cm) }\end{array}$ & $\begin{array}{l}\text { Percentage } \\
\text { of sample } \\
\text { examined }\end{array}$ & $\begin{array}{l}\text { Number of } \\
\text { P. atlanticum }\end{array}$ & $\begin{array}{c}\text { Number of } \\
\text { E. vitrea }\end{array}$ & $\begin{array}{l}\text { Number of } \\
\text { B. morgani }\end{array}$ & $\begin{array}{c}\text { Number of } \\
\text { other } \\
\text { foraminifers }\end{array}$ & $\begin{array}{l}\text { Total } \\
\text { number of } \\
\text { foraminifers } \\
\text { (N) }\end{array}$ & $\begin{array}{l}\text { Number of } \\
\text { foraminifers } \\
\text { per gram }\end{array}$ & $\begin{array}{l}\text { Percentage } \\
\text { of PEB } \\
\text { species }\end{array}$ & $\begin{array}{c}\text { Confidence } \\
\text { interval } \\
\text { of PEB }\end{array}$ \\
\hline 1.5 & 25.00 & 25 & 0 & 0 & 360 & 385 & 134.27 & 8.57 & 2.80 \\
\hline 14.5 & 12.50 & 24 & 1 & 0 & 334 & 359 & 174.58 & 6.96 & 2.63 \\
\hline 22.5 & 12.50 & 14 & 2 & 0 & 408 & 424 & 289.23 & 3.77 & 1.81 \\
\hline 30.5 & 25.00 & 46 & 1 & 0 & 732 & 779 & 178.59 & 6.03 & 1.67 \\
\hline 44.5 & 25.00 & 22 & 1 & 0 & 413 & 436 & 121.25 & 5.28 & 2.10 \\
\hline 50.5 & 25.00 & 22 & 1 & 0 & 509 & 532 & 99.18 & 6.58 & 2.11 \\
\hline
\end{tabular}


Table 4. Benthic foraminiferal data from core MRD05-6BC, Louisiana Shelf, Gulf of Mexico.

\begin{tabular}{|c|c|c|c|c|c|c|c|c|c|}
\hline $\begin{array}{l}\text { Sample depth } \\
\text { midpoint } \\
(\mathrm{cm})\end{array}$ & $\begin{array}{l}\text { Percentage } \\
\text { of sample } \\
\text { examined }\end{array}$ & $\begin{array}{l}\text { Number of } \\
\text { P. atlanticum }\end{array}$ & $\begin{array}{c}\text { Number of } \\
\text { E. vitrea }\end{array}$ & $\begin{array}{l}\text { Number of } \\
\text { B. morgani }\end{array}$ & $\begin{array}{c}\text { Number of } \\
\text { other } \\
\text { foraminifers }\end{array}$ & $\begin{array}{l}\text { Total number of } \\
\text { foraminifers } \\
\text { (N) }\end{array}$ & $\begin{array}{l}\text { Number of } \\
\text { foraminifers } \\
\text { per gram }\end{array}$ & $\begin{array}{l}\text { Percentage } \\
\text { of PEB } \\
\text { species }\end{array}$ & $\begin{array}{c}\text { Confidence } \\
\text { interval } \\
\text { of PEB }\end{array}$ \\
\hline 0.5 & 40.63 & 45 & 4 & 3 & 303 & 355 & 130.33 & 14.65 & 3.68 \\
\hline 1.5 & 12.50 & 68 & 8 & 1 & 272 & 349 & 416.42 & 22.06 & 4.35 \\
\hline 2.5 & 75.00 & 53 & 4 & 2 & 281 & 340 & 67.61 & 17.35 & 4.03 \\
\hline 3.5 & 50.00 & 64 & 5 & 2 & 325 & 396 & 118.12 & 17.93 & 3.78 \\
\hline 4.5 & 20.00 & 53 & 10 & 2 & 364 & 429 & 319.92 & 15.15 & 3.39 \\
\hline 5.5 & 100.00 & 48 & 2 & 3 & 294 & 347 & 51.75 & 15.27 & 3.79 \\
\hline 6.5 & 20.00 & 59 & 17 & 4 & 292 & 372 & 277.41 & 21.51 & 4.18 \\
\hline 7.5 & 25.00 & 72 & 13 & 2 & 338 & 425 & 253.55 & 20.47 & 3.84 \\
\hline 8.5 & 12.50 & 60 & 18 & 5 & 280 & 363 & 433.12 & 22.87 & 4.32 \\
\hline 9.5 & 18.75 & 49 & 11 & 3 & 338 & 401 & 318.97 & 15.71 & 3.56 \\
\hline 10.5 & 6.25 & 47 & 5 & 0 & 261 & 313 & 746.92 & 16.61 & 4.12 \\
\hline 11.5 & 12.50 & 47 & 6 & 2 & 285 & 340 & 405.68 & 16.18 & 3.91 \\
\hline 12.5 & 16.67 & 39 & 20 & 6 & 347 & 412 & 368.69 & 15.78 & 3.52 \\
\hline 13.5 & 25.00 & 45 & 10 & 2 & 270 & 327 & 195.08 & 17.43 & 4.11 \\
\hline 14.5 & 12.50 & 29 & 12 & 3 & 289 & 333 & 397.32 & 13.21 & 3.64 \\
\hline 15.5 & 15.63 & 36 & 5 & 3 & 325 & 369 & 352.22 & 11.92 & 3.31 \\
\hline 16.5 & 6.25 & 24 & 12 & 5 & 272 & 313 & 746.92 & 13.10 & 3.74 \\
\hline 17.5 & 12.50 & 30 & 11 & 3 & 309 & 353 & 421.19 & 12.46 & 3.45 \\
\hline 18.5 & 12.50 & 12 & 6 & 0 & 295 & 313 & 373.46 & 5.75 & 2.58 \\
\hline 19.5 & 12.50 & 15 & 13 & 2 & 302 & 332 & 396.13 & 9.04 & 3.08 \\
\hline 20.5 & 12.50 & 22 & 15 & 1 & 356 & 394 & 470.11 & 9.64 & 2.91 \\
\hline 21.5 & 18.75 & 20 & 5 & 1 & 345 & 371 & 295.11 & 7.01 & 2.60 \\
\hline 22.5 & 25.00 & 18 & 0 & 0 & 326 & 344 & 205.22 & 5.23 & 2.35 \\
\hline 23.5 & 12.50 & 17 & 4 & 0 & 306 & 327 & 390.17 & 6.42 & 2.66 \\
\hline 24.5 & 12.50 & 34 & 2 & 1 & 344 & 381 & 454.60 & 9.71 & 2.97 \\
\hline 25.5 & 12.50 & 19 & 5 & 1 & 286 & 311 & 371.08 & 8.04 & 3.02 \\
\hline 26.5 & 12.50 & 11 & 6 & 3 & 291 & 311 & 371.08 & 6.43 & 2.73 \\
\hline 27.5 & 18.75 & 31 & 3 & 1 & 351 & 386 & 307.04 & 9.07 & 2.86 \\
\hline 28.5 & 12.50 & 23 & 11 & 2 & 337 & 373 & 445.05 & 9.65 & 3.00 \\
\hline 29.5 & 18.75 & 26 & 3 & 2 & 354 & 385 & 306.25 & 8.05 & 2.72 \\
\hline 30.5 & 9.38 & 32 & 9 & 1 & 395 & 437 & 695.22 & 9.61 & 2.76 \\
\hline 31.5 & 6.25 & 16 & 7 & 1 & 338 & 362 & 863.85 & 6.63 & 2.56 \\
\hline 32.5 & 8.33 & 11 & 3 & 2 & 349 & 365 & 653.26 & 4.38 & 2.10 \\
\hline 33.5 & 6.25 & 12 & 4 & 3 & 314 & 333 & 794.65 & 5.71 & 2.49 \\
\hline 34.5 & 12.50 & 14 & 2 & 5 & 364 & 385 & 459.37 & 5.45 & 2.27 \\
\hline 35.5 & 25.00 & 64 & 40 & 4 & 698 & 806 & 480.85 & 13.40 & 2.35 \\
\hline 36.5 & 25.00 & 25 & 4 & 3 & 473 & 505 & 301.27 & 6.34 & 2.12 \\
\hline 37.5 & 9.38 & 10 & 8 & 1 & 292 & 311 & 494.77 & 6.11 & 2.66 \\
\hline 38.5 & 6.25 & 20 & 21 & 0 & 281 & 322 & 768.40 & 12.73 & 3.64 \\
\hline 39.5 & 9.38 & 12 & 6 & 2 & 312 & 332 & 528.18 & 6.02 & 2.56 \\
\hline 40.5 & 25.00 & 46 & 7 & 0 & 757 & 810 & 483.23 & 6.54 & 1.70 \\
\hline 41.5 & 37.50 & 47 & 16 & 1 & 701 & 765 & 304.26 & 8.37 & 1.96 \\
\hline
\end{tabular}


Table 5. Benthic foraminiferal data from core MRD05-6GC, Louisiana Shelf, Gulf of Mexico.

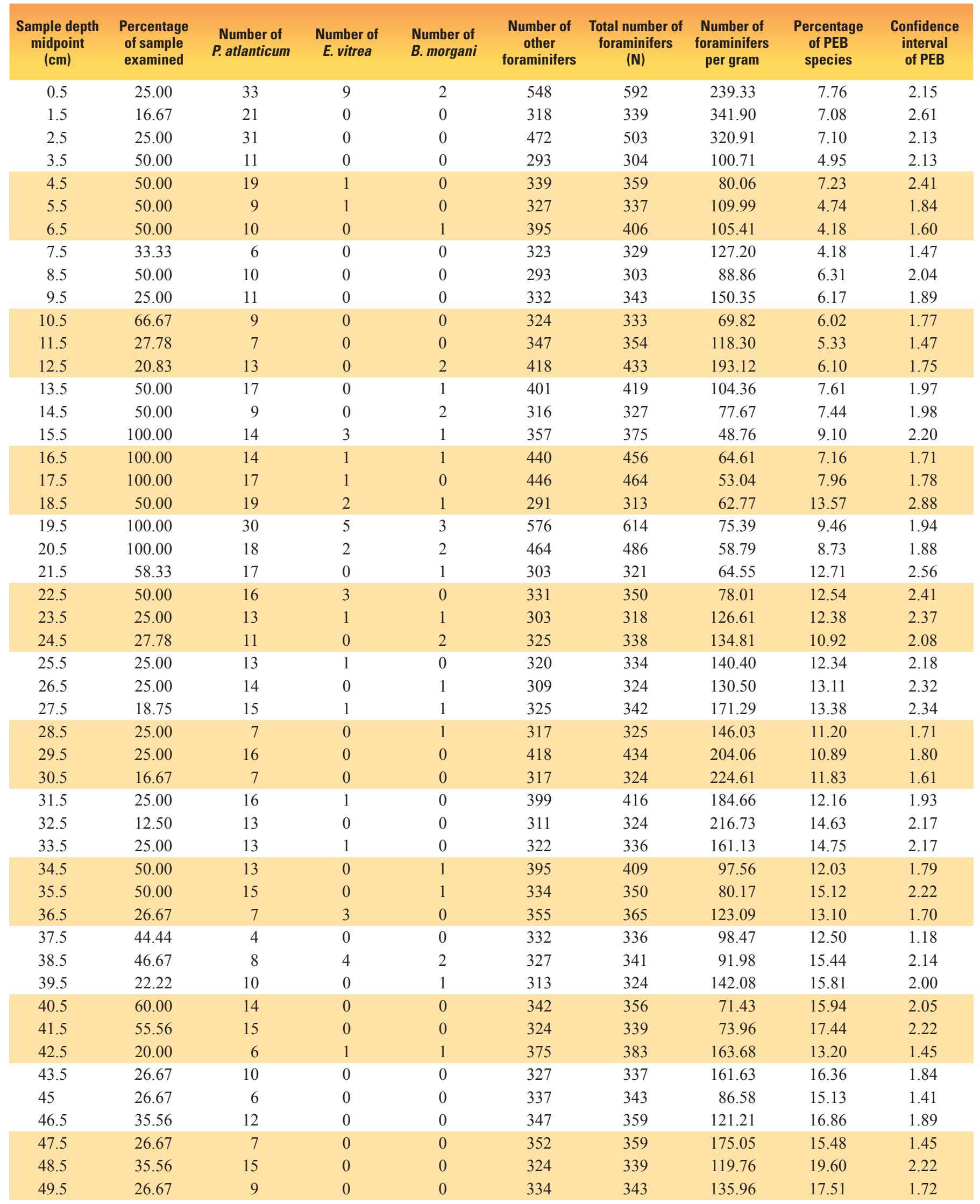


Table 5. (Continued) Benthic foraminiferal data from core MRD05-6GC, Louisiana Shelf, Gulf of Mexico.

\begin{tabular}{|c|c|c|c|c|c|c|c|c|c|}
\hline $\begin{array}{l}\text { Sample depth } \\
\text { midpoint } \\
\text { (cm) }\end{array}$ & $\begin{array}{l}\text { Percentage } \\
\text { of sample } \\
\text { examined }\end{array}$ & $\begin{array}{l}\text { Number of } \\
\text { P. atlanticum }\end{array}$ & $\begin{array}{l}\text { Number of } \\
\text { E. vitrea }\end{array}$ & $\begin{array}{l}\text { Number of } \\
\text { B. morgani }\end{array}$ & $\begin{array}{l}\text { Number of } \\
\text { other } \\
\text { foraminifers }\end{array}$ & $\begin{array}{l}\text { Total number of } \\
\text { foraminifers } \\
\text { (N) }\end{array}$ & $\begin{array}{c}\text { Number of } \\
\text { foraminifers } \\
\text { per gram }\end{array}$ & $\begin{array}{l}\text { Percentage } \\
\text { of PEB } \\
\text { species }\end{array}$ & $\begin{array}{l}\text { Confidence } \\
\text { interval } \\
\text { of PEB }\end{array}$ \\
\hline 50.5 & 20.00 & 10 & 1 & 0 & 346 & 357 & 188.47 & 17.77 & 1.82 \\
\hline 51.5 & 20.00 & 10 & 1 & 0 & 340 & 351 & 153.42 & 18.38 & 1.85 \\
\hline 52.5 & 22.22 & 11 & 0 & 1 & 340 & 352 & 131.91 & 18.68 & 1.92 \\
\hline 53.5 & 26.67 & 16 & 0 & 0 & 343 & 359 & 110.94 & 20.26 & 2.17 \\
\hline 54.5 & 26.67 & 8 & 3 & 0 & 318 & 329 & 114.66 & 20.60 & 1.97 \\
\hline 55.5 & 20.00 & 7 & 1 & 0 & 344 & 352 & 144.69 & 18.46 & 1.58 \\
\hline 56.5 & 26.67 & 13 & 0 & 1 & 330 & 344 & 131.18 & 21.06 & 2.12 \\
\hline 57.5 & 28.89 & 10 & 0 & 0 & 326 & 336 & 103.87 & 20.71 & 1.84 \\
\hline 59 & 11.11 & 10 & 0 & 0 & 318 & 328 & 175.13 & 21.70 & 1.89 \\
\hline 60.5 & 25.00 & 8 & 0 & 0 & 307 & 315 & 124.28 & 22.31 & 1.76 \\
\hline 61.5 & 25.00 & 10 & 0 & 1 & 364 & 375 & 147.80 & 19.64 & 1.73 \\
\hline 62.5 & 25.00 & 2 & 0 & 1 & 309 & 312 & 135.73 & 20.87 & 1.10 \\
\hline 63.5 & 50.00 & 10 & 0 & 0 & 319 & 329 & 73.23 & 23.04 & 1.88 \\
\hline 64.5 & 25.00 & 8 & 0 & 0 & 347 & 355 & 141.09 & 20.89 & 1.57 \\
\hline 65.5 & 25.00 & 12 & 0 & 0 & 309 & 321 & 150.02 & 25.08 & 2.11 \\
\hline 66.5 & 18.75 & 9 & 0 & 0 & 341 & 350 & 176.44 & 22.14 & 1.68 \\
\hline 67.5 & 12.50 & 6 & 0 & 0 & 356 & 362 & 251.18 & 20.65 & 1.34 \\
\hline 68.5 & 9.38 & 11 & 0 & 0 & 302 & 313 & 271.55 & 26.32 & 2.07 \\
\hline 69.5 & 18.75 & 10 & 0 & 0 & 366 & 376 & 210.19 & 21.72 & 1.65 \\
\hline 70.5 & 50.00 & 14 & 0 & 1 & 428 & 443 & 91.55 & 19.74 & 1.71 \\
\hline 71.5 & 100.00 & 4 & 1 & 0 & 141 & 146 & 29.46 & 54.26 & 3.00 \\
\hline 72.5 & 100.00 & 2 & 0 & 0 & 221 & 223 & 31.56 & 33.71 & 1.26 \\
\hline 73.5 & 100.00 & 8 & 0 & 0 & 220 & 228 & 30.60 & 37.05 & 2.42 \\
\hline 74.5 & 66.67 & 15 & 0 & 0 & 310 & 325 & 66.62 & 28.87 & 2.32 \\
\hline 75.5 & 25.00 & 23 & 0 & 0 & 370 & 393 & 162.32 & 26.62 & 2.36 \\
\hline 76.5 & 50.00 & 17 & 0 & 0 & 301 & 318 & 66.05 & 31.06 & 2.51 \\
\hline 77.5 & 40.00 & 7 & 0 & 0 & 379 & 386 & 98.80 & 22.30 & 1.35 \\
\hline 78.5 & 31.25 & 7 & 1 & 0 & 318 & 326 & 101.14 & 27.20 & 1.71 \\
\hline 79.5 & 50.00 & 14 & 0 & 0 & 327 & 341 & 74.10 & 28.59 & 2.14 \\
\hline 80.5 & 41.67 & 12 & 0 & 0 & 370 & 382 & 84.94 & 25.00 & 1.78 \\
\hline 81.5 & 35.71 & 6 & 0 & 0 & 314 & 320 & 93.91 & 27.87 & 1.51 \\
\hline 82.5 & 33.33 & 14 & 0 & 0 & 311 & 325 & 89.57 & 31.03 & 2.24 \\
\hline 83.5 & 50.00 & 7 & 0 & 0 & 327 & 334 & 78.09 & 27.68 & 1.56 \\
\hline 84.5 & 33.33 & 19 & 0 & 0 & 302 & 321 & 105.90 & 34.27 & 2.62 \\
\hline 85.5 & 50.00 & 26 & 0 & 0 & 322 & 348 & 83.38 & 34.63 & 2.80 \\
\hline 86.5 & 50.00 & 27 & 0 & 0 & 302 & 329 & 84.54 & 37.58 & 3.01 \\
\hline 87.5 & 50.00 & 23 & 0 & 0 & 356 & 379 & 84.24 & 31.04 & 2.44 \\
\hline 88.5 & 33.33 & 34 & 0 & 0 & 362 & 396 & 127.19 & 33.84 & 2.80 \\
\hline 89.5 & 25.00 & 27 & 0 & 1 & 302 & 330 & 143.52 & 38.58 & 3.05 \\
\hline 90.5 & 27.27 & 20 & 0 & 3 & 302 & 325 & 112.59 & 36.59 & 2.83 \\
\hline 91.5 & 35.00 & 11 & 0 & 0 & 301 & 312 & 107.43 & 34.05 & 2.08 \\
\hline 92.5 & 20.00 & 20 & 1 & 1 & 347 & 369 & 152.31 & 32.71 & 2.45 \\
\hline 93.5 & 15.00 & 16 & 0 & 0 & 333 & 349 & 184.72 & 32.88 & 2.23 \\
\hline 94.5 & 25.00 & 12 & 0 & 0 & 300 & 312 & 142.62 & 35.50 & 2.17 \\
\hline 95.5 & 18.75 & 15 & 0 & 0 & 350 & 365 & 174.14 & 31.57 & 2.07 \\
\hline 96.5 & 25.00 & 15 & 2 & 1 & 324 & 342 & 127.77 & 35.03 & 2.40 \\
\hline 97.5 & 25.00 & 17 & 1 & 1 & 287 & 306 & 141.22 & 40.24 & 2.75 \\
\hline 98.5 & 31.25 & 21 & 1 & 1 & 301 & 324 & 100.96 & 40.03 & 2.84 \\
\hline
\end{tabular}


Table 5. (Continued) Benthic foraminiferal data from core MRD05-6GC, Louisiana Shelf, Gulf of Mexico.

\begin{tabular}{|c|c|c|c|c|c|c|c|c|c|}
\hline $\begin{array}{l}\text { Sample depth } \\
\text { midpoint } \\
\text { (cm) }\end{array}$ & $\begin{array}{l}\text { Percentage } \\
\text { of sample } \\
\text { examined }\end{array}$ & $\begin{array}{l}\text { Number of } \\
P . \text { atlanticum }\end{array}$ & $\begin{array}{l}\text { Number of } \\
\text { E. vitrea }\end{array}$ & $\begin{array}{l}\text { Number of } \\
\text { B. morgani }\end{array}$ & $\begin{array}{c}\text { Number of } \\
\text { other } \\
\text { foraminifers }\end{array}$ & $\begin{array}{l}\text { Total number of } \\
\text { foraminifers } \\
\text { (N) }\end{array}$ & $\begin{array}{c}\text { Number of } \\
\text { foraminifers } \\
\text { per gram }\end{array}$ & $\begin{array}{l}\text { Percentage } \\
\text { of PEB } \\
\text { species }\end{array}$ & $\begin{array}{l}\text { Confidence } \\
\text { interval } \\
\text { of PEB }\end{array}$ \\
\hline 99.5 & 50.00 & 16 & 0 & 0 & 304 & 320 & 75.52 & 37.99 & 2.42 \\
\hline 100.5 & 40.00 & 17 & 0 & 0 & 334 & 351 & 102.80 & 35.18 & 2.28 \\
\hline 101.5 & 31.11 & 16 & 0 & 0 & 321 & 337 & 101.60 & 36.60 & 2.31 \\
\hline 102.5 & 60.00 & 18 & 0 & 0 & 363 & 381 & 71.51 & 33.20 & 2.16 \\
\hline 103.5 & 48.89 & 10 & 0 & 0 & 331 & 341 & 75.83 & 34.29 & 1.82 \\
\hline 104.5 & 35.56 & 14 & 0 & 0 & 324 & 338 & 82.64 & 36.57 & 2.16 \\
\hline 105.5 & 40.00 & 20 & 0 & 1 & 390 & 411 & 102.80 & 32.18 & 2.16 \\
\hline 106.5 & 60.00 & 20 & 0 & 0 & 319 & 339 & 67.26 & 39.66 & 2.55 \\
\hline 107.5 & 60.00 & 13 & 0 & 2 & 323 & 338 & 69.01 & 37.31 & 2.23 \\
\hline 108.5 & 40.00 & 13 & 0 & 0 & 329 & 342 & 91.30 & 36.93 & 2.06 \\
\hline 109.5 & 80.00 & 14 & 0 & 0 & 315 & 329 & 48.06 & 39.21 & 2.21 \\
\hline 110.5 & 37.78 & 18 & 0 & 0 & 326 & 344 & 75.00 & 39.42 & 2.39 \\
\hline 111.5 & 42.22 & 15 & 0 & 0 & 318 & 333 & 78.24 & 39.78 & 2.26 \\
\hline 112.5 & 100.00 & 17 & 1 & 0 & 345 & 363 & 42.75 & 37.83 & 2.27 \\
\hline 113.5 & 84.44 & 22 & 0 & 0 & 308 & 330 & 46.95 & 43.99 & 2.73 \\
\hline 114.5 & 64.44 & 16 & 1 & 0 & 313 & 330 & 53.31 & 42.01 & 2.42 \\
\hline 115.5 & 64.44 & 11 & 0 & 0 & 349 & 360 & 61.44 & 36.25 & 1.81 \\
\hline 116.5 & 40.00 & 19 & 0 & 0 & 314 & 333 & 86.96 & 43.15 & 2.53 \\
\hline 117.5 & 40.00 & 11 & 2 & 0 & 337 & 350 & 95.18 & 38.72 & 2.01 \\
\hline 118.5 & 53.33 & 17 & 1 & 1 & 328 & 347 & 72.33 & 41.62 & 2.43 \\
\hline 119.5 & 20.00 & 14 & 2 & 1 & 348 & 365 & 172.33 & 38.94 & 2.19 \\
\hline 120.5 & 42.22 & 20 & 1 & 1 & 369 & 391 & 94.14 & 38.35 & 2.32 \\
\hline 121.5 & 28.89 & 12 & 0 & 0 & 338 & 350 & 100.90 & 39.50 & 1.94 \\
\hline 122.5 & 46.67 & 13 & 0 & 1 & 314 & 328 & 85.27 & 43.15 & 2.22 \\
\hline 123.5 & 24.44 & 19 & 0 & 0 & 320 & 339 & 123.16 & 44.53 & 2.49 \\
\hline 124.5 & 31.11 & 19 & 0 & 1 & 307 & 327 & 108.25 & 46.74 & 2.64 \\
\hline 125.5 & 31.11 & 33 & 0 & 0 & 312 & 345 & 113.21 & 50.80 & 3.15 \\
\hline 126.5 & 22.22 & 22 & 0 & 0 & 312 & 334 & 139.11 & 47.60 & 2.70 \\
\hline 127.5 & 48.89 & 17 & 1 & 0 & 332 & 350 & 83.90 & 43.83 & 2.35 \\
\hline 128.5 & 31.11 & 34 & 2 & 1 & 313 & 350 & 102.04 & 52.56 & 3.27 \\
\hline 129.5 & 42.22 & 31 & 2 & 0 & 310 & 343 & 80.63 & 52.42 & 3.17 \\
\hline 130.5 & 31.11 & 29 & 1 & 0 & 307 & 337 & 119.14 & 52.28 & 3.09 \\
\hline 131.5 & 28.89 & 12 & 0 & 0 & 318 & 330 & 126.16 & 45.13 & 2.05 \\
\hline 132.5 & 31.11 & 21 & 0 & 1 & 329 & 351 & 103.55 & 46.66 & 2.57 \\
\hline 133.5 & 22.22 & 14 & 0 & 0 & 354 & 368 & 174.29 & 41.67 & 1.98 \\
\hline 134.5 & 8.89 & 15 & 5 & 1 & 310 & 331 & 317.62 & 49.84 & 2.67 \\
\hline 135.5 & 20.00 & 22 & 0 & 0 & 326 & 348 & 197.61 & 48.31 & 2.60 \\
\hline 136.5 & 100.00 & 159 & 28 & 4 & 1506 & 1697 & 156.69 & 21.48 & 1.53 \\
\hline 137.5 & 15.56 & 23 & 2 & 0 & 318 & 343 & 204.91 & 51.10 & 2.79 \\
\hline 138.5 & 20.00 & 26 & 1 & 1 & 324 & 352 & 173.95 & 51.08 & 2.87 \\
\hline 139.5 & 20.00 & 18 & 1 & 0 & 375 & 394 & 197.41 & 42.27 & 2.15 \\
\hline 140.5 & 28.89 & 23 & 1 & 0 & 330 & 354 & 118.91 & 49.85 & 2.66 \\
\hline 141.5 & 48.89 & 23 & 0 & 0 & 324 & 347 & 79.69 & 50.77 & 2.66 \\
\hline 142.5 & 40.00 & 13 & 0 & 0 & 313 & 326 & 80.65 & 49.68 & 2.16 \\
\hline 143.5 & 40.00 & 23 & 0 & 1 & 371 & 395 & 88.15 & 44.88 & 2.39 \\
\hline 144.5 & 40.00 & 17 & 0 & 1 & 337 & 355 & 103.02 & 47.92 & 2.32 \\
\hline
\end{tabular}


Table 6. Benthic foraminiferal data from core PE0609BC, Louisiana Shelf, Gulf of Mexico.

\begin{tabular}{|c|c|c|c|c|c|c|c|c|c|}
\hline $\begin{array}{l}\text { Sample depth } \\
\text { midpoint } \\
\text { (cm) }\end{array}$ & $\begin{array}{l}\text { Percentage } \\
\text { of sample } \\
\text { examined }\end{array}$ & $\begin{array}{l}\text { Number of } \\
\text { P. atlanticum }\end{array}$ & $\begin{array}{c}\text { Number of } \\
\text { E. vitrea }\end{array}$ & $\begin{array}{l}\text { Number of } \\
\text { B. morgani }\end{array}$ & $\begin{array}{c}\text { Number of } \\
\text { other } \\
\text { foraminifers }\end{array}$ & $\begin{array}{l}\text { Total number of } \\
\text { foraminifers } \\
\text { (N) }\end{array}$ & $\begin{array}{l}\text { Number of } \\
\text { foraminifers } \\
\text { per gram }\end{array}$ & $\begin{array}{l}\text { Percentage } \\
\text { of PEB } \\
\text { species }\end{array}$ & $\begin{array}{l}\text { Confidence } \\
\text { interval } \\
\text { of PEB }\end{array}$ \\
\hline 1.5 & 3.13 & 50 & 33 & 14 & 237 & 334 & 1126.80 & 29.04 & 4.87 \\
\hline 2.5 & 3.13 & 46 & 25 & 15 & 328 & 414 & 1396.69 & 20.77 & 3.91 \\
\hline 5.5 & 3.13 & 55 & 23 & 15 & 278 & 371 & 1251.62 & 25.07 & 4.41 \\
\hline 6.5 & 4.69 & 45 & 22 & 7 & 261 & 335 & 753.45 & 22.09 & 4.44 \\
\hline 7.5 & 6.25 & 28 & 4 & 2 & 316 & 350 & 590.39 & 9.71 & 3.10 \\
\hline 8.5 & 1.56 & 22 & 0 & 1 & 503 & 526 & 3549.07 & 4.37 & 1.75 \\
\hline 12.5 & 3.91 & 24 & 1 & 0 & 360 & 385 & 1039.08 & 6.49 & 2.46 \\
\hline 13.5 & 7.81 & 30 & 2 & 3 & 360 & 395 & 533.04 & 8.86 & 2.80 \\
\hline 14.5 & 6.25 & 21 & 0 & 1 & 382 & 404 & 681.48 & 5.45 & 2.21 \\
\hline 15.5 & 6.25 & 25 & 0 & 0 & 360 & 385 & 649.43 & 6.49 & 2.46 \\
\hline 16.5 & 12.50 & 17 & 2 & 0 & 421 & 440 & 371.10 & 4.32 & 1.90 \\
\hline 17.5 & 7.81 & 26 & 0 & 1 & 323 & 350 & 472.31 & 7.71 & 2.80 \\
\hline
\end{tabular}


Table 7. Benthic foraminiferal data from core MCH-11BC, Louisiana Shelf, Gulf of Mexico.

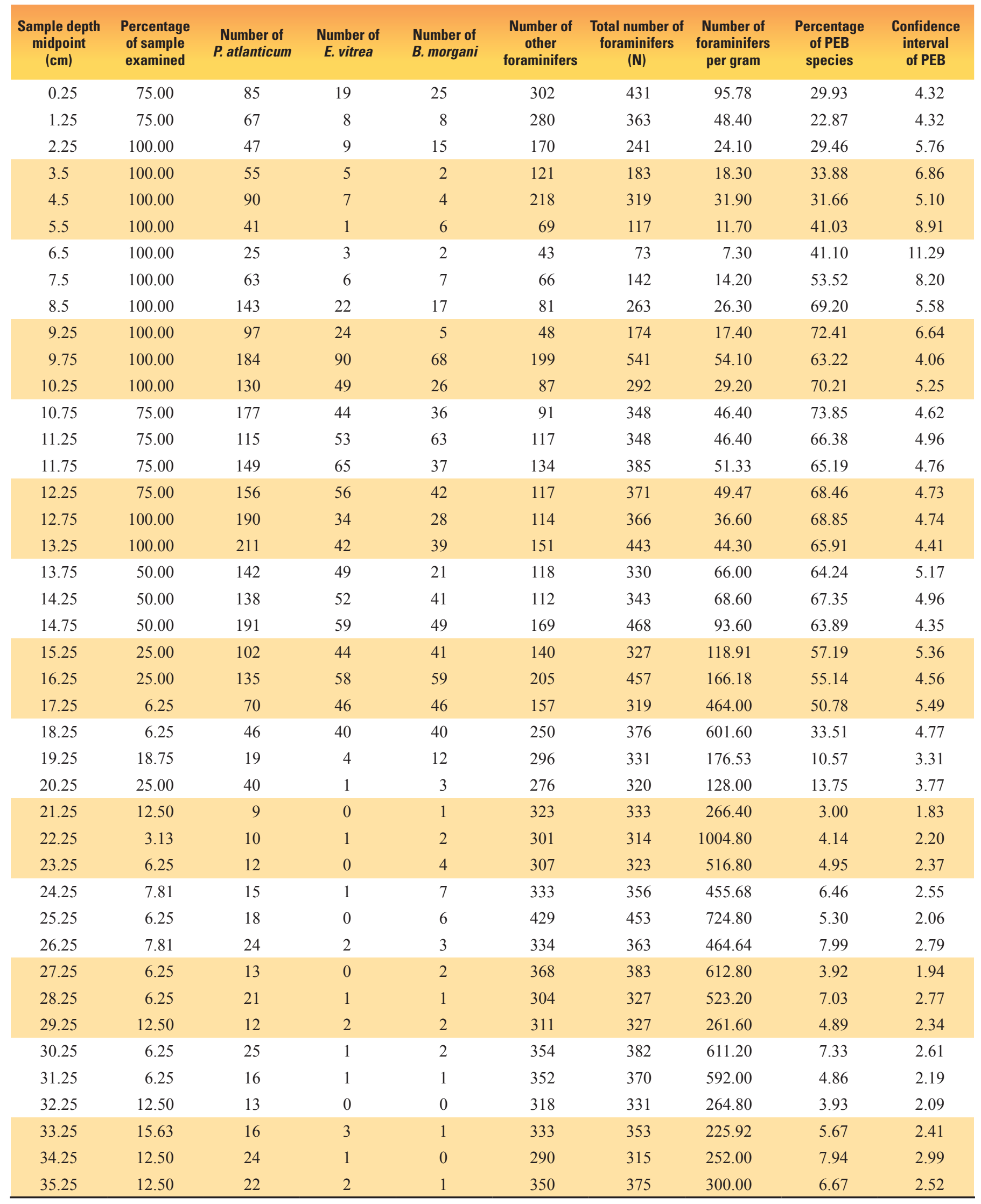


Table 8. Benthic foraminiferal data from core MCH-33BC, Louisiana Shelf, Gulf of Mexico.

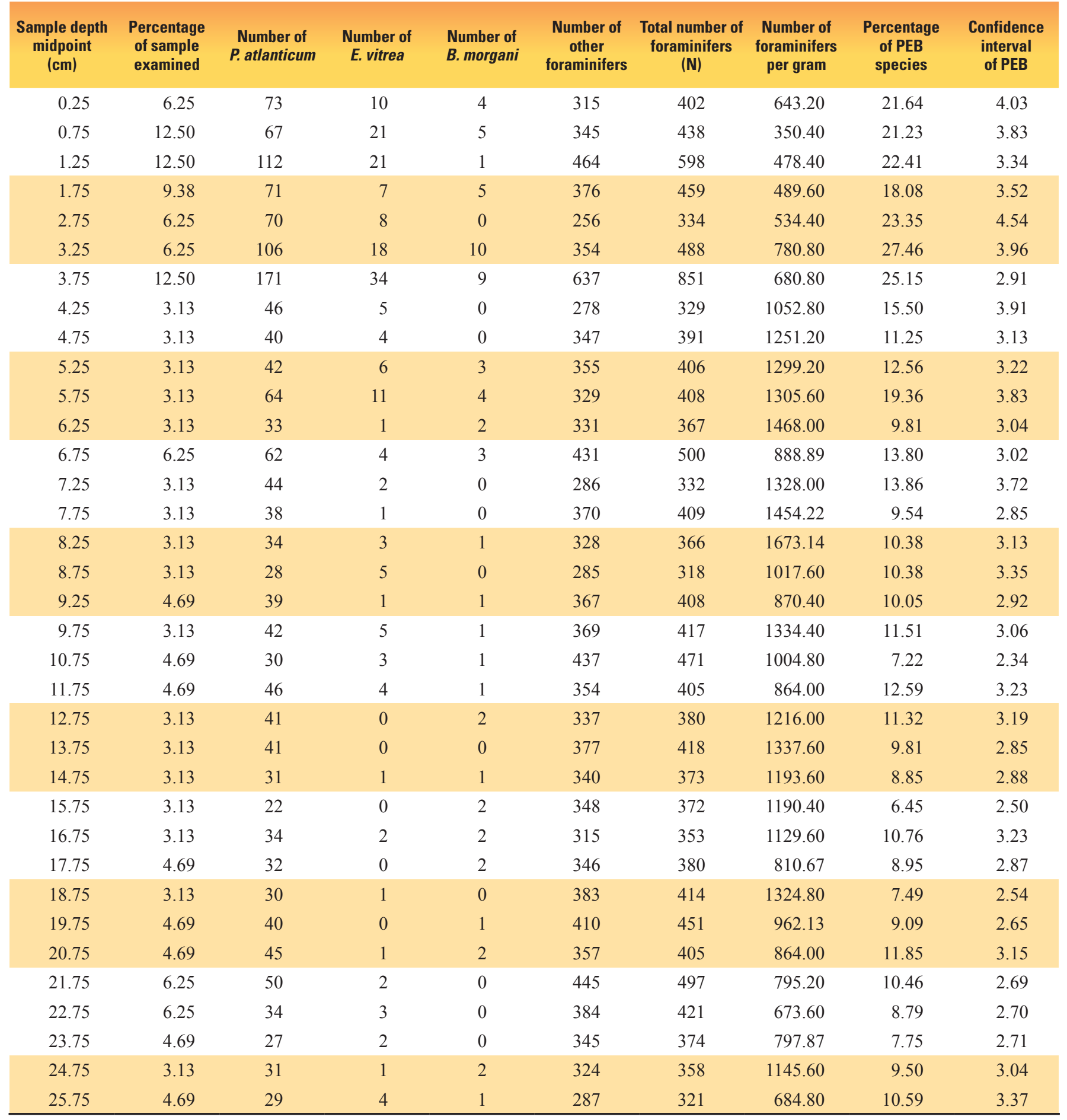


Table 9. Benthic foraminiferal data from core PE07-1BC, Louisiana Shelf, Gulf of Mexico.

\begin{tabular}{|c|c|c|c|c|c|c|c|c|c|}
\hline $\begin{array}{l}\text { Sample depth } \\
\text { midpoint } \\
\text { (cm) }\end{array}$ & $\begin{array}{l}\text { Percentage } \\
\text { of sample } \\
\text { examined }\end{array}$ & $\begin{array}{l}\text { Number of } \\
P . \text { atlanticum }\end{array}$ & $\begin{array}{l}\text { Number of } \\
\text { E. vitrea }\end{array}$ & $\begin{array}{l}\text { Number of } \\
\text { B. morgani }\end{array}$ & $\begin{array}{c}\text { Number of } \\
\text { other } \\
\text { foraminifers }\end{array}$ & $\begin{array}{l}\text { Total number of } \\
\text { foraminifers } \\
\text { (N) }\end{array}$ & $\begin{array}{l}\text { Number of } \\
\text { foraminifers } \\
\text { per gram }\end{array}$ & $\begin{array}{l}\text { Percentage } \\
\text { of PEB } \\
\text { species }\end{array}$ & $\begin{array}{c}\text { Confidence } \\
\text { interval } \\
\text { of PEB }\end{array}$ \\
\hline 0.25 & 75.00 & 44 & 2 & 0 & 372 & 418 & 222.93 & 11.00 & 3.00 \\
\hline 1.25 & 12.50 & 65 & 1 & 0 & 386 & 452 & 361.60 & 14.60 & 3.26 \\
\hline 2.25 & 12.50 & 90 & 4 & 2 & 341 & 437 & 349.60 & 21.97 & 3.88 \\
\hline 3.25 & 12.50 & 55 & 6 & 1 & 374 & 436 & 436.00 & 14.22 & 3.28 \\
\hline 4.25 & 7.81 & 45 & 7 & 8 & 299 & 359 & 459.52 & 16.71 & 3.86 \\
\hline 5.25 & 12.50 & 50 & 12 & 4 & 295 & 361 & 361.00 & 18.28 & 3.99 \\
\hline 6.25 & 31.25 & 47 & 6 & 4 & 324 & 381 & 121.92 & 14.96 & 3.58 \\
\hline 7.25 & 37.50 & 40 & 6 & 4 & 338 & 388 & 147.81 & 12.89 & 3.33 \\
\hline 8.25 & 25.00 & 64 & 11 & 14 & 277 & 366 & 146.40 & 24.32 & 4.40 \\
\hline 9.25 & 25.00 & 35 & 4 & 6 & 260 & 305 & 122.00 & 14.75 & 3.98 \\
\hline 10.25 & 31.25 & 44 & 6 & 12 & 289 & 351 & 112.32 & 17.66 & 3.99 \\
\hline 11.25 & 31.25 & 57 & 4 & 8 & 247 & 316 & 101.12 & 21.84 & 4.56 \\
\hline 12.25 & 9.38 & 21 & 2 & 3 & 303 & 329 & 350.93 & 7.90 & 2.92 \\
\hline 13.25 & 4.69 & 30 & 0 & 0 & 320 & 350 & 746.67 & 8.57 & 2.93 \\
\hline 14.25 & 6.25 & 35 & 0 & 1 & 336 & 372 & 595.20 & 9.68 & 3.00 \\
\hline 15.25 & 1.95 & 9 & 1 & 0 & 314 & 324 & 1658.88 & 3.09 & 1.88 \\
\hline 16.25 & 3.13 & 28 & 2 & 0 & 303 & 333 & 1065.60 & 9.01 & 3.08 \\
\hline 17.25 & 3.13 & 24 & 2 & 0 & 287 & 313 & 1001.60 & 8.31 & 3.06 \\
\hline 18.25 & 3.91 & 28 & 1 & 0 & 313 & 342 & 972.80 & 8.48 & 2.95 \\
\hline 19.25 & 3.13 & 20 & 1 & 1 & 315 & 337 & 1078.40 & 6.53 & 2.64 \\
\hline 20.25 & 3.91 & 25 & 1 & 2 & 326 & 354 & 906.24 & 7.91 & 2.81 \\
\hline 21.25 & 3.13 & 16 & 1 & 2 & 301 & 320 & 1024.00 & 5.94 & 2.59 \\
\hline 22.25 & 2.34 & 16 & 0 & 1 & 290 & 307 & 1309.87 & 5.54 & 2.56 \\
\hline 23.25 & 3.13 & 23 & 3 & 1 & 323 & 350 & 1120.00 & 7.71 & 2.80 \\
\hline 24.25 & 3.13 & 16 & 0 & 0 & 272 & 288 & 921.60 & 5.56 & 2.65 \\
\hline 25.25 & 3.13 & 18 & 0 & 0 & 319 & 337 & 1078.40 & 5.34 & 2.40 \\
\hline 26.25 & 3.13 & 19 & 1 & 1 & 329 & 350 & 1493.33 & 6.00 & 2.49 \\
\hline 27.25 & 3.13 & 27 & 0 & 0 & 352 & 379 & 1617.07 & 7.12 & 2.59 \\
\hline 28.25 & 3.13 & 23 & 0 & 0 & 388 & 411 & 1315.20 & 5.60 & 2.22 \\
\hline 29.25 & 3.13 & 27 & 0 & 1 & 318 & 346 & 1107.20 & 8.09 & 2.87 \\
\hline 30.25 & 4.69 & 19 & 0 & 0 & 392 & 411 & 974.22 & 4.62 & 2.03 \\
\hline 31.25 & 3.13 & 34 & 0 & 0 & 326 & 360 & 1152.00 & 9.44 & 3.02 \\
\hline 32.25 & 1.56 & 17 & 1 & 2 & 250 & 270 & 1728.00 & 7.41 & 3.12 \\
\hline 33.25 & 3.13 & 26 & 1 & 0 & 403 & 430 & 1376.00 & 6.28 & 2.29 \\
\hline 34.25 & 3.13 & 30 & 0 & 1 & 343 & 374 & 1196.80 & 8.29 & 2.79 \\
\hline 35.25 & 3.13 & 24 & 0 & 1 & 276 & 301 & 963.20 & 8.31 & 3.12 \\
\hline 36.25 & 1.56 & 26 & 2 & 0 & 288 & 316 & 2022.40 & 8.86 & 3.13 \\
\hline 37.25 & 1.56 & 37 & 1 & 0 & 299 & 337 & 2156.80 & 11.28 & 3.38 \\
\hline
\end{tabular}


Table 10. Benthic foraminiferal data from core PE07-7BC, Louisiana Shelf, Gulf of Mexico.

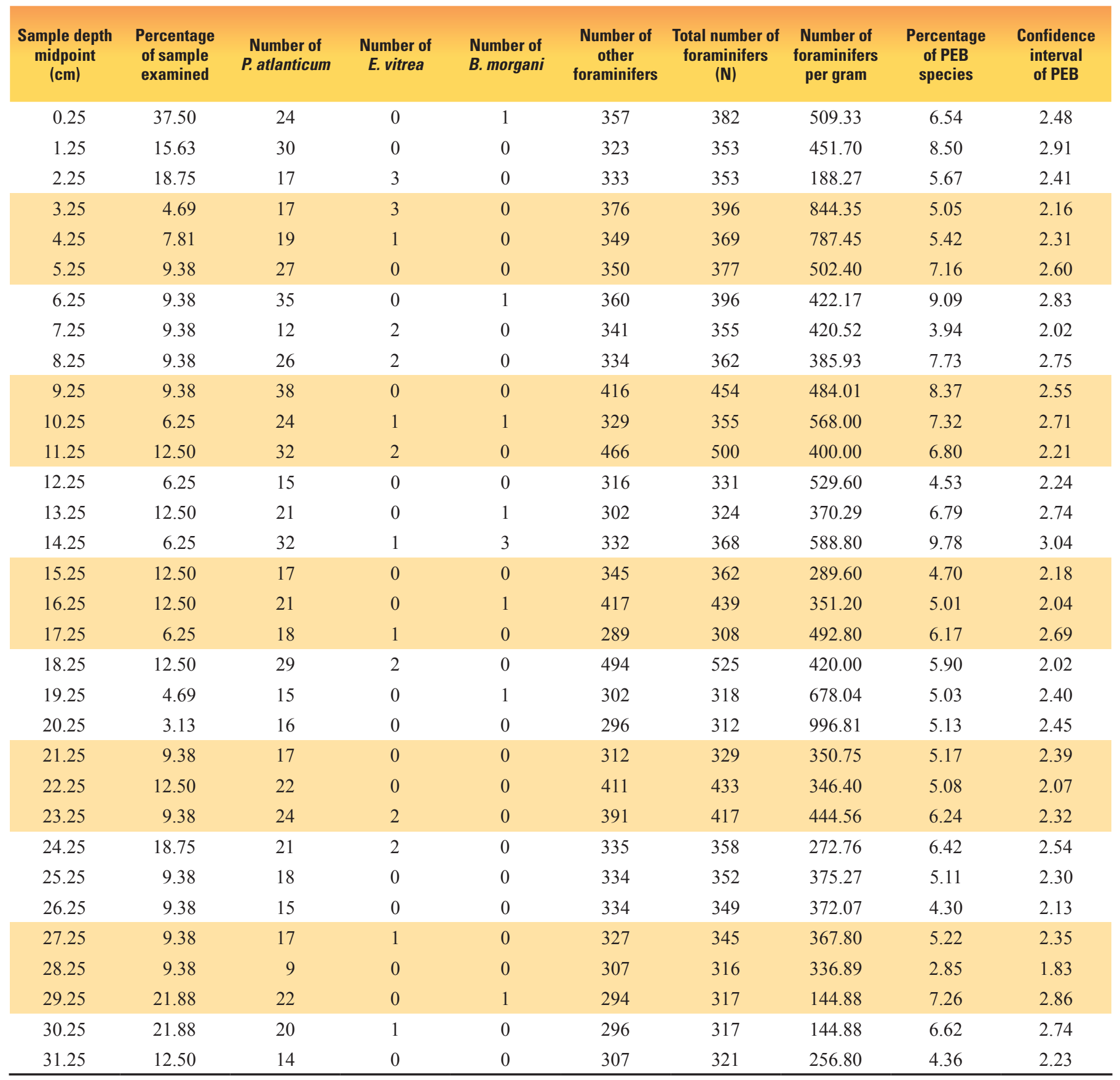


Table 11. Benthic foraminiferal data from core PE07-8BC, Louisiana Shelf, Gulf of Mexico.

\begin{tabular}{|c|c|c|c|c|c|c|c|c|c|}
\hline $\begin{array}{l}\text { Sample depth } \\
\text { midpoint } \\
\text { (cm) }\end{array}$ & $\begin{array}{l}\text { Percentage } \\
\text { of sample } \\
\text { examined }\end{array}$ & $\begin{array}{c}\text { Number of } \\
P . \text { atlanticum }\end{array}$ & $\begin{array}{l}\text { Number of } \\
\text { E. vitrea }\end{array}$ & $\begin{array}{l}\text { Number of } \\
\text { B. morgani }\end{array}$ & $\begin{array}{c}\text { Number of } \\
\text { other } \\
\text { foraminifers }\end{array}$ & $\begin{array}{l}\text { Total number of } \\
\text { foraminifers } \\
\text { (N) }\end{array}$ & $\begin{array}{l}\text { Number of } \\
\text { foraminifers } \\
\text { per gram }\end{array}$ & $\begin{array}{l}\text { Percentage } \\
\text { of PEB } \\
\text { species }\end{array}$ & $\begin{array}{l}\text { Confidence } \\
\text { interval } \\
\text { of PEB }\end{array}$ \\
\hline 0.25 & 50.00 & 197 & 15 & 1 & 368 & 581 & 232.40 & 36.66 & 3.92 \\
\hline 1.25 & 25.00 & 148 & 10 & 6 & 167 & 331 & 132.40 & 49.55 & 5.39 \\
\hline 2.25 & 18.75 & 171 & 9 & 5 & 266 & 451 & 240.53 & 41.02 & 4.54 \\
\hline 3.25 & 25.00 & 108 & 19 & 7 & 210 & 344 & 137.60 & 38.95 & 5.15 \\
\hline 4.25 & 25.00 & 107 & 18 & 8 & 188 & 321 & 128.40 & 41.43 & 5.39 \\
\hline 5.25 & 25.00 & 150 & 18 & 4 & 209 & 381 & 152.40 & 45.14 & 5.00 \\
\hline 6.25 & 12.50 & 97 & 39 & 29 & 217 & 382 & 305.60 & 43.19 & 4.97 \\
\hline 7.25 & 12.50 & 108 & 40 & 35 & 333 & 516 & 412.80 & 35.47 & 4.13 \\
\hline 8.25 & 18.75 & 112 & 36 & 36 & 258 & 442 & 235.73 & 41.63 & 4.60 \\
\hline 9.25 & 6.25 & 115 & 31 & 33 & 264 & 443 & 708.80 & 40.41 & 4.57 \\
\hline 10.25 & 12.50 & 96 & 16 & 24 & 217 & 353 & 282.40 & 38.53 & 5.08 \\
\hline 11.25 & 12.50 & 95 & 22 & 27 & 242 & 386 & 308.80 & 37.31 & 4.82 \\
\hline 11.75 & 6.25 & 74 & 16 & 19 & 197 & 306 & 489.60 & 35.62 & 5.37 \\
\hline 12.25 & 9.38 & 75 & 22 & 22 & 255 & 374 & 398.72 & 31.82 & 4.72 \\
\hline 12.75 & 12.50 & 132 & 26 & 30 & 303 & 491 & 392.80 & 38.29 & 4.30 \\
\hline 13.25 & 5.66 & 96 & 24 & 18 & 265 & 403 & 712.01 & 34.24 & 4.63 \\
\hline 13.75 & 12.50 & 129 & 45 & 31 & 337 & 542 & 433.60 & 37.82 & 4.08 \\
\hline 14.25 & 11.52 & 99 & 32 & 19 & 293 & 443 & 384.55 & 33.86 & 4.41 \\
\hline 14.75 & 9.38 & 93 & 21 & 20 & 277 & 411 & 438.40 & 32.60 & 4.53 \\
\hline 15.25 & 6.25 & 74 & 21 & 15 & 236 & 346 & 553.60 & 31.79 & 4.91 \\
\hline 16.25 & 6.25 & 78 & 15 & 16 & 275 & 384 & 614.40 & 28.39 & 4.51 \\
\hline 16.75 & 9.38 & 65 & 6 & 7 & 259 & 337 & 359.47 & 23.15 & 4.50 \\
\hline 17.25 & 6.25 & 74 & 5 & 5 & 261 & 345 & 552.00 & 24.35 & 4.53 \\
\hline 17.75 & 9.38 & 75 & 10 & 16 & 286 & 387 & 412.80 & 26.10 & 4.38 \\
\hline 18.25 & 9.38 & 82 & 11 & 10 & 289 & 392 & 417.91 & 26.28 & 4.36 \\
\hline 18.75 & 6.25 & 81 & 9 & 15 & 220 & 325 & 520.00 & 32.31 & 5.08 \\
\hline 19.25 & 6.25 & 92 & 13 & 20 & 326 & 451 & 721.60 & 27.72 & 4.13 \\
\hline 19.75 & 6.25 & 69 & 8 & 10 & 203 & 290 & 464.00 & 30.00 & 5.27 \\
\hline 20.25 & 15.63 & 95 & 10 & 13 & 247 & 365 & 233.53 & 32.33 & 4.80 \\
\hline 20.75 & 6.25 & 129 & 12 & 17 & 270 & 428 & 684.80 & 36.92 & 4.57 \\
\hline 21.25 & 4.69 & 87 & 15 & 12 & 202 & 316 & 674.13 & 36.08 & 5.29 \\
\hline 22.25 & 3.13 & 127 & 24 & 15 & 324 & 490 & 1568.00 & 33.88 & 4.19 \\
\hline 23.25 & 12.50 & 154 & 10 & 15 & 374 & 553 & 491.56 & 32.37 & 3.90 \\
\hline 24.25 & 6.25 & 98 & 19 & 20 & 339 & 476 & 1088.00 & 28.78 & 4.07 \\
\hline 25.25 & 3.13 & 50 & 23 & 13 & 244 & 330 & 1320.00 & 26.06 & 4.74 \\
\hline 26.25 & 3.13 & 51 & 14 & 11 & 280 & 356 & 1139.20 & 21.35 & 4.26 \\
\hline 27.25 & 3.13 & 52 & 8 & 13 & 370 & 443 & 1417.60 & 16.48 & 3.45 \\
\hline 28.25 & 6.25 & 38 & 10 & 9 & 322 & 379 & 606.40 & 15.04 & 3.60 \\
\hline 29.25 & 4.69 & 42 & 9 & 10 & 314 & 375 & 800.00 & 16.27 & 3.74 \\
\hline 30.25 & 4.69 & 30 & 2 & 5 & 278 & 315 & 672.00 & 11.75 & 3.56 \\
\hline 31.25 & 4.69 & 51 & 6 & 4 & 345 & 406 & 866.13 & 15.02 & 3.48 \\
\hline 32.25 & 3.13 & 29 & 2 & 5 & 280 & 316 & 1011.20 & 11.39 & 3.50 \\
\hline 33.25 & 3.13 & 33 & 3 & 8 & 293 & 337 & 1078.40 & 13.06 & 3.60 \\
\hline 34.25 & 6.25 & 37 & 2 & 6 & 320 & 365 & 584.00 & 12.33 & 3.37 \\
\hline 35.25 & 9.38 & 50 & 1 & 4 & 256 & 311 & 473.90 & 17.68 & 4.24 \\
\hline 36.25 & 6.25 & 39 & 0 & 2 & 278 & 319 & 510.40 & 12.85 & 3.67 \\
\hline 37.25 & 6.25 & 46 & 4 & 10 & 362 & 422 & 675.20 & 14.22 & 3.33 \\
\hline 38.25 & 4.69 & 22 & 2 & 10 & 276 & 310 & 661.33 & 10.97 & 3.48 \\
\hline 39.25 & 4.69 & 38 & 1 & 7 & 329 & 375 & 800.00 & 12.27 & 3.32 \\
\hline 40.25 & 6.25 & 27 & 2 & 9 & 269 & 307 & 491.20 & 12.38 & 3.68 \\
\hline 41.25 & 6.25 & 36 & 6 & 5 & 340 & 387 & 619.20 & 12.14 & 3.25 \\
\hline
\end{tabular}




\section{Faunal Analysis}

Osterman (2003) determined that in the benthic foraminiferal assemblages found in the Gulf of Mexico, the relative abundance of three low-oxygen-tolerant species was highest in the Louisiana hypoxic zone. Therefore, in this research, only these three species were identified and counted as described herein. Processed samples contained few to abundant benthic foraminifera $(30-2,186$ foraminifers/g or $7-1,658$ foraminifers $/ \mathrm{cm}^{3}$ ). When required, a representative subsample of approximately 300 specimens was obtained for faunal analysis using a microsplitter. Benthic foraminifers were picked from the $>125-\mu \mathrm{m}$ faunal split spread across a 45 square, holepunched tray and viewed through a binocular stereo microscope. When an individual foraminifer was encountered in the picking process, it was identified and counted as one of four categories (P. atlanticum, E. vitrea, B. morgani, and others). This allowed for the calculation of the cumulative percentage of these three species in each sample. After identification and counting, each specimen was dropped though a hole in the punched tray onto a stationary 60 -square micropaleontological slide placed in a cardboard cutout. All faunal slides will be curated at the Natural History Museum, Smithsonian Institution, at the conclusion of the study. Identifications of benthic foraminiferal species were based on the use of classic and modern literature, including the taxonomy of Phleger and Parker (1951), Bandy (1954), Parker (1954), Anderson (1961), Bock and others (1971), Murray (1971, 2000), Poag (1981), Morkhoven and others (1986), and Loeblich and Tappan $(1988,1994)$. Tables 2-11 display sample interval, percentage of sample examined, benthic foraminiferal counts, relative percentage of the PEB species (Osterman, 2003), and confidence-interval data (Patterson and Fishbein, 1989) for gravity- and box-core samples.

\section{Acknowledgments}

Many thanks to Peter Swarzenski (USGS), David Senn (Harvard School of Public Health) and Steve DiMarco (Texas A\&M University) for having graciously collected core samples for this research. Thanks to Jackie Smith, Katie Merriweather, Ashley Daigle, and Amy Spaziani for their help in processing these and many more samples during the last 5 years. Finally, many thanks to Caitlin Nay for her excellent help with the preparation of this report.

\section{References Cited}

Anderson, H.V., 1961, Genesis and paleontology of the Mississippi River mudlumps, Pt. 2: Foraminifera of the mudlumps, Lower Mississippi River Delta: Department of Conservation, Louisiana Geological Survey, Geological Bulletin 35, pt. 2, 208 p.

Bandy, O.L., 1954, Distribution of some shallow water foraminifera in the Gulf of Mexico: U.S. Geological Survey Professional Paper 254-F, p. 125-140.

Blackwelder, P., Hood, T., Alvarez-Zarikian, C., Nelsen, T.A., and McKee, B., 1996, Benthic foraminifera from the NECOP study area impacted by the Mississippi River plume and seasonal hypoxia: Quaternary International, v. 31, p. 19-36.

Bock, W.D., Lynts, G.W., Smith, S., Wright, R., Hay, W.W., and Jones, J.I., 1971, A symposium of recent south Florida foraminifera: Memoir 1: Miami Geological Society, 245 p.

Goolsby, D.A., Battaglin, W.A., Aulenbach, B.T., and Hooper, R.P., 2001, Nitrogen input to the Gulf of Mexico: Journal of Environmental Quality, v. 30, p. 329-336.

Loeblich, A.R., Jr., and Tappan, H., 1988, Foraminiferal genera and their classification: New York, Van Nostrand Reinhold, 970 p., 847 plates.

Loeblich, A.R., Jr., and Tappan, H., 1994, Foraminifera of the Sahul Shelf and Timor Sea: Cushman Foundation for Foraminiferal Research, Special Publication 31, 661 p.

Morkhoven, F.P.C.M., Berggren, W.A., and Edwards, A.S., 1986, Cenozoic cosmopolitan deep-water benthic foraminifera: Bulletin des Centres de Recherches ExplorationProduction, Elf Aquitaine, Memoir 11, Pau, France, 421 p.

Murray, J.W., 1971, An atlas of British recent foraminiferids: New York, Elsevier, 244 p.

Murray, J.W., 2000, Revised taxonomy: An atlas of British recent foraminiferids: Journal of Micropaleontology, v. 19, p. 44.

Osterman, L.E., 2003, Benthic foraminifers from the Continental Shelf and Slope of the Gulf of Mexico: An indicator of shelf hypoxia: Estuarine, Coastal, and Shelf Science, v. 58, p. $17-35$.

Osterman, L.E., Poore, R.Z., and Swarzenski, P.W., 2008a, The last 1000 years of natural and anthropogenic lowoxygen bottom-water on the Louisiana Shelf, Gulf of Mexico: Marine Micropaleontology, v. 66, p. 291-303.

Osterman, L.E., Poore, R.Z., and Swarzenski, P.W., 2008b, Gulf of Mexico Dead Zone-The 100-year record: U.S. Geological Survey Open-File Report 2008-1099, 2 p. 
Osterman, L.E., Poore, R.Z., Swarzenski, P.W., and Turner, R.E., 2005, Reconstructing an 180-yr record of natural and anthropogenic induced hypoxia from the sediments of the Louisiana Continental Shelf: Geology, v. 33, p. 329-332.

Parker, F.L., 1954, Distribution of the foraminifera in the northeastern Gulf of Mexico: Bulletin of the Museum of Comparative Zoology, v. 111, no. 10, p. 454-547.

Patterson, R.T., and Fishbein, E., 1989, Re-examination of the statistical methods used to determine the number of point counts needed for micropaleontological quantitative research: Journal of Paleontology, v. 63, p. 245-248.

Phleger, F.B., and Parker, F.L., 1951, Foraminiferal species: Geological Society America Memoir 46, Pt. 2, 64 p.

Poag, C.W., 1981, Ecological atlas of benthic foraminifera of the Gulf of Mexico: Woods Hole, MA, Marine Science International, $174 \mathrm{p}$.

Rabalais, N.N., 2002, Nitrogen in aquatic systems: Ambios, v. 31, p. 102-112.

Rabalais, N.N., and Turner, R.E., 2001, Hypoxia in the northern Gulf of Mexico: Description, causes and change, in Rabalais, N.N., and Turner, R.E., eds., Coastal hypoxia: Consequences for living resources and ecosystems: Washington, D.C., Coastal and Estuaries Studies, American Geophysical Union, v. 58, p. 1-36.

Rabalais, N.N., Turner, R.E., Justic', D., Dortch, Q., and Wiseman, W.J., Jr., 1999, Characterization of hypoxia: Topic 1-Report for the integrated assessment on hypoxia in the Gulf of Mexico: Silver Spring, MD, NOAA Coastal Ocean Program Decision Analysis Series No. 15, 67 p.

Rabalais, N.N., Turner, R.E., Justic', D., Dortch, Q., Wiseman, W.J., Jr., and Sen Gupta, B.K., 1996, Nutrient changes in the Mississippi River and the system responses on the adjacent Continental Shelf: Estuaries, v. 19, p. 386-407.

Rabalais, N.N., Wiseman, W.J., Jr., and Turner, R.E., 1994, Comparison of continuous records of near-bottom oxygen from the hypoxia zone along the Louisiana coast: Estuaries, v. 17, p. $850-861$.

Sen Gupta, B.K., Turner, R.E., and Rabalais, N.N., 1996, Seasonal oxygen depletion in Continental-Shelf waters of Louisiana: Historical record of benthic foraminifers: Geology, v. 24, p. 227-230.

Swarzenski, P.W., Campbell, P.L., Osterman, L.E., and Poore, R.Z., 2008, A 1000-year sediment record of recurring hypoxia off the Mississippi River: The potential role of terrestrially derived organic matter inputs: Marine Chemistry, v. 109, p. 130-142.

Turner, R.E., Rabalais, N.N., Swenson, E.M., Kasprzak, M., and Romaire, T., 2005, Summer hypoxia, Northern Gulf of Mexico: 1978 to 1995: Marine Environmental Research, v. 59 , p. $65-77$.

\section{Faunal Reference List}

\section{Buliminella morgani}

Anderson, H.V., 1961, Genesis and paleontology of the Mississippi River mudlumps, Pt. 2: Foraminifera of the mudlumps, Lower Mississippi River Delta: Department of Conservation, Louisiana Geological Survey, Geological Bulletin 35, pt. 2, p. 87, pl. 19, fig. 10.

\section{Epistominella vitrea}

Parker, In Parker, F.L., Phleger, F.B., and Pierson, J.F., Ecology of foraminifera from San Antonio Bay and environs, Southwest Texas. Cushman Foundation for Foraminiferal Research, Special Publication 2, p. 9, pl. 4, figs. 34, 35, 36, 40 , and 41.

\section{Pseudononion atlanticum (Cushman) = Nonionella atlantica}

Cushman, J.A. 1947. New species and varieties of foraminifera from off the southeastern coast of the united States: Contributions to the Cushman Laboratory for Foraminiferal Research, v. 23, p. 11, pl. 5, figs. 21-22. 

